\title{
Four-electron quantum dot in a magnetic field
}

\author{
M. B. Tavernier, ${ }^{1 * *}$ E. Anisimovas, ${ }^{1, \dagger}$ F. M. Peeters, ${ }^{1, \dagger}$ B. Szafran, ${ }^{1,2}$ J. Adamowski, ${ }^{2}$ and S. Bednarek ${ }^{2}$ \\ ${ }^{1}$ Departement Natuurkunde, Universiteit Antwerpen (Campus Drie Eiken), B-2610 Antwerpen, Belgium \\ ${ }^{2}$ Faculty of Physics and Nuclear Techniques, AGH University of Science and Technology, Kraków, Poland
}

(Received 23 June 2003; published 12 November 2003)

\begin{abstract}
We present a theoretical treatment of four two-dimensional electrons in a harmonic confinement potential in the presence of an external magnetic field using the exact diagonalization approach. The ground state properties and the spin and angular momentum transitions for different electron interaction strengths and magnetic fields are obtained. A magnetic field-confinement strength phase diagram is presented indicating a rich variety of ground states. An interesting feature of this system is the depolarization of spins by application of a magnetic field. The results are compared to several approximate theories.
\end{abstract}

DOI: 10.1103/PhysRevB.68.205305

PACS number(s): 73.21.La, 71.10.-w

\section{INTRODUCTION}

The rapid progress in experimental techniques has made it possible to construct few electron quantum dots in semiconductor materials (for recent reviews see Refs. 1-3). These dots contain a few electrons trapped in a plane and laterally confined by an external potential. Some of their properties, such as the electronic shell structure, for example, closely resemble those of real atoms, however, in quantum dots the experimental control and tunability of the involved parameters is much broader. This makes quantum dots an ideal system to study electron-electron interactions and correlation effects. The nature of the confining potential may be different depending on the experimental setup. In most cases, however, a harmonic model is appropriate to describe this confinement. ${ }^{4}$ The competition between the interelectron repulsion, confinement, and spin effects leads to interesting physics, such as transformations of the multielectron ground state as a function of magnetic field, in particular, the transition from a Fermi liquid to a Wigner crystal $^{5,6}$ and switching of the spin and the angular momentum of the system. ${ }^{7,8}$

Among the computational approaches, a special role is played by exact diagonalizations (ED's). ${ }^{6,8-13}$ Especially useful in the limit of small particle number, these calculations are capable of providing accurate results against which reliability and accuracy of approximate methods can be gauged. Most of the ED-based work concentrated in the limit of strong magnetic fields where the role of electron-electron correlations is particularly important. Moreover, in this limit the numerical effort is greatly reduced by restricting the single-particle basis to the lowest (often also spin polarized) Landau level (LL). ${ }^{10}$ Recently this work has been extending into the regime of zero and intermediate magnetic fields. Mikhailov and co-workers studied a system of three interacting electrons in zero ${ }^{14}$ and finite ${ }^{15}$ magnetic fields. At low magnetic fields this system exhibits an interesting ground state transition at from the $(L, S)=(1,1 / 2)$ state to the $(0,3 / 2)$ state, here $L$ the total angular momentum and $S$ the total spin of the system. This transition is accompanied by a change in electron structure, from an isosceles to an equilateral triangle. The degenerate ground states of a four-electron system without magnetic field was also considered ${ }^{16}$ and no ground state transition was found. Earlier, six-electron quan- tum dot in zero magnetic field was studied ${ }^{6}$ at several discrete values of interaction strength (density).

In the present paper we report ED calculations of fourelectron quantum dot in a broad range of magnetic fields. The obtained results are compared with those obtained by several approximate methods, namely, renormalized perturbation series, ${ }^{17,18}$ multicenter Hartree-Fock (MCHF) ${ }^{19}$ and a restricted Hartree-Fock (RHF) algorithm. We look for possible magnetic-field-induced ground state angular momentum and spin transitions. Particular attention is paid to the polarization and breakdown of the maximum density droplet state (MDD) in which all electron occupy the lowest orbitals of the lowest LL.

The structure of the paper is as follows. In Sec. II the theory of the involved computational methods is presented, ED results are described in Sec. III and compared to other methods in Sec. IV, and in Sec. V the conclusions are given.

\section{THEORY}

We consider a system of four electrons which are restricted to move in the $z=0$ plane and are laterally trapped in a harmonic confining potential, $V(r)=m^{*} \omega_{0}^{2} r^{2} / 2$, where $m^{*}$ is the effective mass of the electrons in the host semiconductor, $\omega_{0}$ is the oscillator frequency of the confining potential, and $\mathbf{r}=(x, y)$ denotes the position in the $z=0$ plane. The Hamiltonian of this system in the presence of a homogeneous magnetic field perpendicular to the $z=0$ plane described using the symmetric gauge $\mathbf{A}=B(-y, x, 0) / 2$ reads

$$
\begin{aligned}
\hat{H}= & \sum_{i=1}^{4}\left(\frac{\hat{\mathbf{p}}_{i}^{2}}{2 m^{*}}+\frac{m^{*} \omega_{0}^{2} r_{i}^{2}}{2}\right)+\sum_{i>j}^{4} \frac{e^{2} / \varepsilon}{\left|\mathbf{r}_{i}-\mathbf{r}_{j}\right|}+\sum_{i=1}^{4} \frac{1}{8} m^{*} \omega_{c}^{2} r_{i}^{2} \\
& +\frac{1}{2} \hbar \omega_{c} \hat{L}_{z}+\frac{1}{2} g^{*} \frac{m^{*}}{m_{e}} \hat{S}_{z} \hbar \omega_{c},
\end{aligned}
$$

where the last three terms represent the contribution due to the magnetic field with $\omega_{c}$ being the cyclotron frequency, $g^{*}$ the effective $g$ factor, and $m_{e}$ the bare electron mass.

The study of this system can be greatly simplified by using dimensionless parameters, i.e., measuring lengths in $l_{0}$ $=\sqrt{\hbar / m^{*} \omega_{0}}$, the oscillator length, and energies in $\hbar \omega_{0}$. A dimensionless parameter $\lambda=l_{0} / a_{B}^{*}$ (here $a_{B}^{*}=\varepsilon \hbar^{2} / e^{2} m^{*}$ is 
the effective Bohr radius) describes the strength of the electron-electron interaction. Large $\lambda$ implies strong interaction and/or a large quantum dot. Since both $\hat{L}_{z}$ and $\hat{S}_{z}$ commute with the Hamiltonian we can perform the calculations separately in subspaces of given $L_{z}$ and $S_{z}$. The dimensionless Hamiltonian becomes

$$
\begin{aligned}
\hat{H}= & \sum_{i=1}^{4}\left[-\frac{1}{2} \nabla_{i}^{2}+\frac{1}{2}\left(1+\frac{1}{4} \Omega_{c}^{2}\right) r_{i}^{2}\right]+\lambda \sum_{i>j}^{4} \frac{1}{\left|\mathbf{r}_{i}-\mathbf{r}_{j}\right|} \\
& +\frac{\Omega_{c}}{2}\left(L_{z}+g * \frac{m^{*}}{m_{e}} S_{z}\right),
\end{aligned}
$$

with $\Omega_{c}=\omega_{c} / \omega_{0}$. In the limit of large $\lambda$ the system becomes classical. $^{20}$

One can easily see that the effect of a magnetic field leads to a rescaling of $\mathbf{r}_{i}$ to $\alpha \mathbf{r}_{i}$, with $\alpha^{4}=1+\frac{1}{4} \Omega_{c}^{2}$. This results in a new effective ${ }^{17} \lambda^{*}=\lambda / \alpha$. Eventually we obtain

$$
\begin{aligned}
\hat{H}= & \alpha^{2}\left[\sum_{i=1}^{4}\left(-\frac{1}{2} \nabla_{i}^{2}+\frac{1}{2} r_{i}^{2}\right)+\lambda * \sum_{i>j}^{4} \frac{1}{\left|\mathbf{r}_{i}-\mathbf{r}_{j}\right|}\right] \\
& +\frac{\Omega_{c}}{2}\left(L_{z}+g * \frac{m^{*}}{m_{e}} S_{z}\right) .
\end{aligned}
$$

The last term which is proportional to the magnetic field involves only the good quantum numbers $L_{z}$ and $S_{z}$ and not the operators themselves, therefore, its effect can be trivially included when the remaining part of the Hamiltonian (3) is diagonalized. That is, one merely needs to solve the problem without magnetic field but with a modified $\lambda \rightarrow \lambda^{*}$. We also note that in this way the magnetic field allows one to continuously change the effective electron-electron interaction. The four-particle wave function is also obtained by rescaling the zero magnetic field result

$$
\Psi_{B \neq 0}\left(\mathbf{r}_{1}, \mathbf{r}_{2}, \mathbf{r}_{3}, \mathbf{r}_{4}\right)=\alpha \Psi_{B=0}\left(\alpha \mathbf{r}_{1}, \alpha \mathbf{r}_{2}, \alpha \mathbf{r}_{3}, \alpha \mathbf{r}_{4}\right)
$$

We use the Fock-Darwin orbitals $\varphi_{n l}(\mathbf{r})$ to construct a complete set of single particle states $\phi_{n l s}(\mathbf{r}, \sigma)$ $=\varphi_{n l}(\mathbf{r}) \chi_{s}(\sigma)$, with $\chi_{s}(\sigma)$ the spin part of the wave function. In dimensionless units these orbitals are given by

$$
\varphi_{n l}(\mathbf{r})=\sqrt{\frac{n !}{\pi(n+|l|) !}} r^{|l|} e^{i l \theta-r^{2} / 2} L_{n}^{|l|}\left(r^{2}\right)
$$

where $L_{n}^{l}(x)$ is the Laguerre polynomial. The energy of these states is independent of $s$ and is a function of two quantum numbers $n$ and $l: E_{n l s}=2 n+|l|+1$ in units of $\hbar \omega_{0}$, where $n$ is the radial quantum number and $l$ the angular quantum number. We use these single particle wave functions to construct Slater determinants

$$
\Psi_{\alpha \beta \gamma \delta}=\frac{1}{\sqrt{4 !}}\left|\begin{array}{cccc}
\phi_{\alpha}\left(\mathbf{r}_{1}, \sigma_{1}\right) & \phi_{\beta}\left(\mathbf{r}_{1}, \sigma_{1}\right) & \phi_{\gamma}\left(\mathbf{r}_{1}, \sigma_{1}\right) & \phi_{\delta}\left(\mathbf{r}_{1}, \sigma_{1}\right) \\
\phi_{\alpha}\left(\mathbf{r}_{2}, \sigma_{2}\right) & \phi_{\beta}\left(\mathbf{r}_{2}, \sigma_{2}\right) & \phi_{\gamma}\left(\mathbf{r}_{2}, \sigma_{2}\right) & \phi_{\delta}\left(\mathbf{r}_{2}, \sigma_{2}\right) \\
\phi_{\alpha}\left(\mathbf{r}_{3}, \sigma_{3}\right) & \phi_{\beta}\left(\mathbf{r}_{3}, \sigma_{3}\right) & \phi_{\gamma}\left(\mathbf{r}_{3}, \sigma_{3}\right) & \phi_{\delta}\left(\mathbf{r}_{3}, \sigma_{3}\right) \\
\phi_{\alpha}\left(\mathbf{r}_{4}, \sigma_{4}\right) & \phi_{\beta}\left(\mathbf{r}_{4}, \sigma_{4}\right) & \phi_{\gamma}\left(\mathbf{r}_{4}, \sigma_{4}\right) & \phi_{\delta}\left(\mathbf{r}_{4}, \sigma_{4}\right)
\end{array}\right|,
$$

with $\alpha=\left(n_{1}, l_{1}, s_{1}\right), \beta=\left(n_{2}, l_{2}, s_{2}\right), \ldots$. The four-particle wave function is expanded in the Slater determinants (6)

$$
\Psi=\sum_{j} C_{j} \Psi_{j}
$$

where $j$ runs over all possible $(\alpha \beta \gamma \delta)$. Inserting the multiparticle wave function (7) into the Schrödinger equation $\hat{H} \Psi=E \Psi$ and multiplying from the left with $\Psi_{i}$ one obtains

$$
\sum_{j}\left(H_{i j}-E \delta_{i j}\right) C_{j}=0
$$

with $H_{i j}=\left\langle\Psi_{i}|\hat{H}| \Psi_{j}\right\rangle$. This eigenvalue equation is solved numerically and yields the eigenenergies and a set of coefficients $C_{j}$ which are used to calculate the four-particle wave function (7).

The accuracy of the results depends on the number of Slater determinants included in the basis (7). In our calculations we include all Slater determinants for which the sum of four single-electron energies is less or equal to some thresh- old energy $E_{\text {th }}$. Increasing $E_{\text {th }}$ will make the results converge to their correct value and make the results reliable for to higher $\lambda$. We were able to obtain a reasonable relative accuracy of approximately $2 \times 10^{-4}$ for $\lambda \leqslant 2$. For example, for the $(14,2)$ state we need 19774 Slater determinants $\left(E_{\mathrm{th}}\right.$ $=26$ ) to obtain a relative accuracy of $5 \times 10^{-5}$.

The exact solutions of the Schrödinger equation for the four-electron system are compared with the results of two different Hartree-Fock approaches: multicenter Hartree-Fock (MCHF) and restricted Hartree-Fock (RHF) calculations. MCHF (Ref. 19) is a method dedicated to confined electron systems at high magnetic fields. Landau gauge and the total spin polarization of the electron system are assumed. The basis set for the four single-electron wave functions consists of four wave functions

$$
\begin{aligned}
\psi_{\mu}(\mathbf{r})= & (a / 2 \pi)^{1 / 2} \sum_{k=1}^{4} c_{k}^{\mu} \exp \left\{-(a / 4)\left[\left(x-X_{k}\right)^{2}+\left(y-Y_{k}\right)^{2}\right]\right. \\
& \left.+(i b / 2)\left(x-X_{k}\right)\left(y+Y_{k}\right)\right\}
\end{aligned}
$$


In the four-electron system the centers of electron localization $\left(X_{k}, Y_{k}\right)$ form a square, the size of which is optimized variationally. Moreover, $a$ and $b$ are also used as nonlinear variational parameters. Basis set functions (9) for fixed $a$ $=b=e B / \hbar$ are the lowest LL eigenfunctions. The applicability of the MCHF is limited to systems, in which the electrons occupy the lowest Fock-Darwin band, i.e., to the MDD and states appearing at higher magnetic fields (Wigner molecules). The MCHF method produces an approximately cylindrically symmetric MDD with a characteristic flat maximum of the charge density. For higher magnetic fields the MCHF method yields a Wigner-molecule phase, in which the electrons are spatially separated. The transformation from the MDD to Wigner phase appears abruptly at a certain magnetic field. This transformation is associated with cusps (discontinuity of the derivative with respect to the magnetic field) of several quantities (interaction energy, radius of the charge density, etc.). At high magnetic fields the charge-density distributions corresponding to the basis functions of MCHF tend to point charges. Therefore, the MCHF reproduces the classical limit of the electron localization at the infinite magnetic field, and at high magnetic fields it provides nearly exact values of the energy.

In the RHF method we assume that the one-electron wave functions are eigenfunctions of the single-electron angular momentum operator. By this assumption the angular correlation between the electrons is totally neglected and only radial wave functions are optimized to minimize the total energy of the confined systems. On the other hand the RHF solutions, in contrary to the MCHF, possess a definite total angular momentum which can be compared with the results of the exact calculations. The RHF used in the present paper solves the HF equations with the finite-difference approach, in which both the Coulomb and exchange potentials are integrated numerically. In this way we avoid possible problems with the finite size of the variational basis. The electronelectron correlation effects are defined as those overlooked by the RHF method. Therefore, the comparison of the exact solution and the RHF results allows us to estimate the role of electron-electron correlation in the four-electron system as function of the electron-electron interaction strength and the external magnetic field.

\section{RESULTS}

\section{A. Ground state phase diagram}

In what follows the four-particle states will be labeled $(L, S)$ where $L$ denotes the total angular momentum and $S$ the total spin of the state. The single particle states will be labeled $(n, l, \uparrow$, or $\downarrow)$ with $n$ and $l$ being the radial and angular quantum numbers, respectively. As a matter of fact, in our convention the states of negative angular momentum are more energetically favorable in a magnetic field. However, for the sake of brevity in the ensuing discussion we will be omitting the "-" sign, that is, the absolute values of $L$ will be given.

We numerically calculated the eigenstates and their corresponding energies as a function of the magnetic field for a fixed value of the interaction constant $\lambda=2.0$. In Fig. 1 we

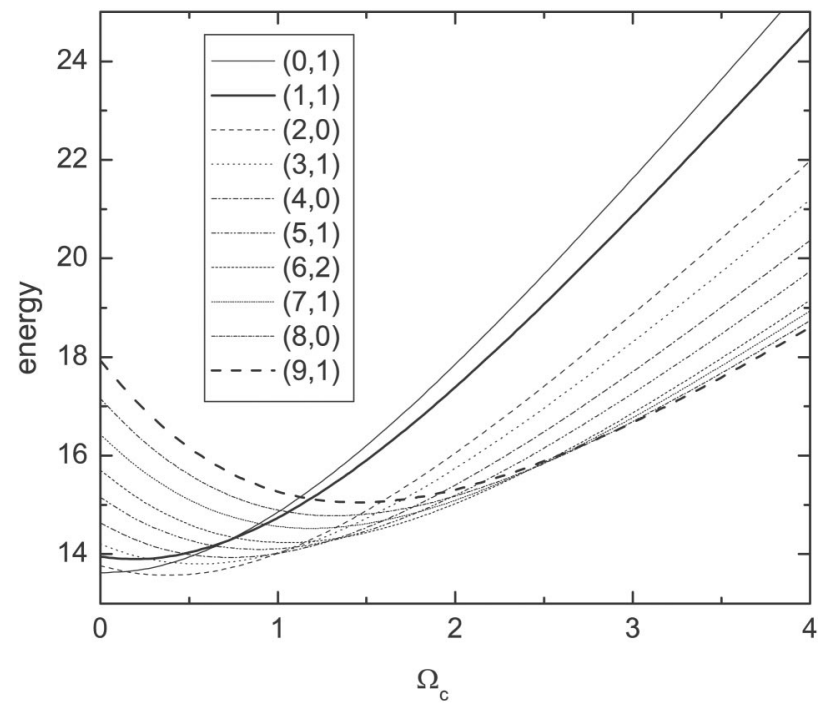

FIG. 1. The energy spectrum as a function of the magnetic field for $\lambda=2.0$. The lowest-energy states of angular momentum up to 9 are shown. The Zeeman energy is included with $g^{*}=-0.44$. The energy is given in units of $\hbar \omega_{0}$. States are labeled $(L, S)$ with $L$ the total angular momentum and $S$ the total spin of the state.

show the evolution of the lowest-energy states for different angular momenta $L$ between 0 and 9 . The spin quantum numbers accompanying the lowest-energy states of all angular momenta are in strict accordance with the predictions of the "magic number" theory ${ }^{21}$ based on the symmetry considerations of four electrons forming a ring. It is interesting to observe that no deviation from this simple rule is found down to the lowest values of the magnetic field, as well as for any $\lambda \leqslant 2$ investigated. In this system the filling factors 2 , $1,1 / 2,1 / 3$ correspond, respectively, to angular momenta $L=2,6,12,18$.

One notes that with increasing magnetic field the terms of ever higher angular momenta are competing to become the ground state resulting in a sequence of angular-momentum transitions. Already at very low fields the ground state $(0,1)$ is succeeded by $(2,0)$, and this transition bears two interesting aspects. First, one observes that the ground-state angular momentum thus increases by 2 , and the state $(1,1)$ is prevented from becoming the ground state. As we will see later, this behavior persists for all values of $\lambda$ and results from the fact that at high magnetic fields the $(1,1)$ state [as well as $(0,1)]$ asymptotically approaches the first excited LL while the $(2,0)$ state corresponds to the lowest LL. As can be seen in Fig. 1 the formation of LL's is apparent already at $\Omega_{c}$ $\approx 2$. Secondly, we note that this transition is a triplet-tosinglet transition, which implies that the application of a magnetic field depolarizes the electron spins. A similar phenomenon was recently found in quantum-dot lithium, ${ }^{15}$ however, in that case such a transition was present only for much larger values of the interaction constant $\lambda \geqslant \lambda_{0}=4.343$, whereas in the present system it is found for all values of $\lambda$, with or without the Zeeman contribution. This transition of the ground state from $(0,1)$ to $(2,0)$ was observed experimentally in Refs. 22,23 by investigating the pairing of conductance peaks in single electron tunneling experiments. 


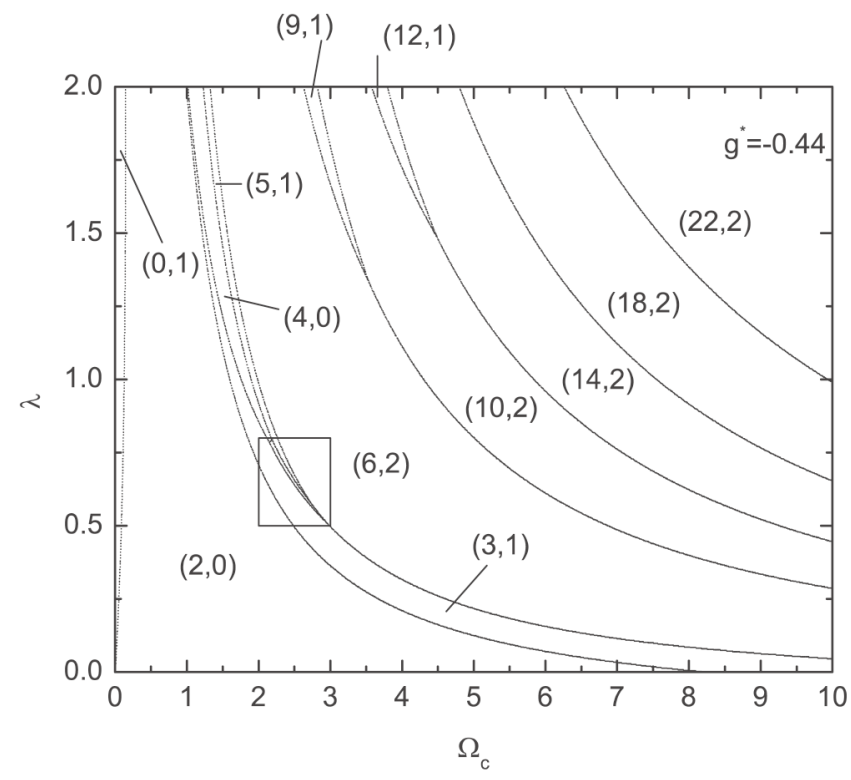

FIG. 2. The ground state configuration as a function of the Coulomb interaction strength and the magnetic field for $g^{*}=-0.44$. Angular momenta up to 22 are included. See Fig. 4 for the magnification of the area inside the rectangle. States are labeled $(L, S)$ with $L$ the total angular momentum and $S$ the total spin of the state.

In order to explore this and other transitions of the ground state in greater detail, we plot the $B-\lambda$ (that is, magnetic field versus interaction) phase diagram in Fig. 2, where the Zeeman contribution is included with $g^{*}=-0.44$. We see that the phase boundary between the two above-discussed states $(0,1)$ and $(2,0)$ starts from the origin. This behavior is readily understood by considering the relevant single-particle configurations. At $\lambda=0.0$ (no interaction) the electrons occupy the four lowest orbitals, i.e., their configuration is $\{(0,0, \uparrow),(0,0, \downarrow),(0,1, \uparrow),(0,-1, \uparrow)\}$. By application of a magnetic field the negative momentum levels will rise in energy while the positive will drop, and this will make the $(0,-1, \uparrow)$ electron jump to the state $(0,1, \downarrow)$. Thus, at $\lambda=0$ the transition from the $(0,1)$ state to the $(2,0)$ state is triggered by an infinitesimally small magnetic field. At finite values of $\lambda$ this jump will be suppressed by the interelectron repulsion up to a certain nonzero value of the magnetic field (on the order of $\Omega_{c} \sim 0.1$ ) when the difference between the single-particle energies of the two levels exceeds the repulsion energy. We stress that the abovedescribed scenario remains unaltered in the presence of Zeeman splitting since the latter increases more slowly with magnetic field than the orbital contribution. Figure 3 shows the corresponding phase diagram when the Zeeman energy is neglected by setting $g^{*}=0$. Here one notices that the states $(2,0),(6,2),(14,0)$, and $(18,2)$ are stable over a longer range than their neighboring states.

One further observes in Fig. 2 that the state $(2,0)$ does not extend to infinitely large magnetic fields in the low $\lambda$ region, but is terminated at $\Omega_{c} \approx 8.17$. In contrast to the previous discussion, this effect is a direct consequence of the Zeeman effect which can be seen from Fig. 3. In the $(2,0)$ state the

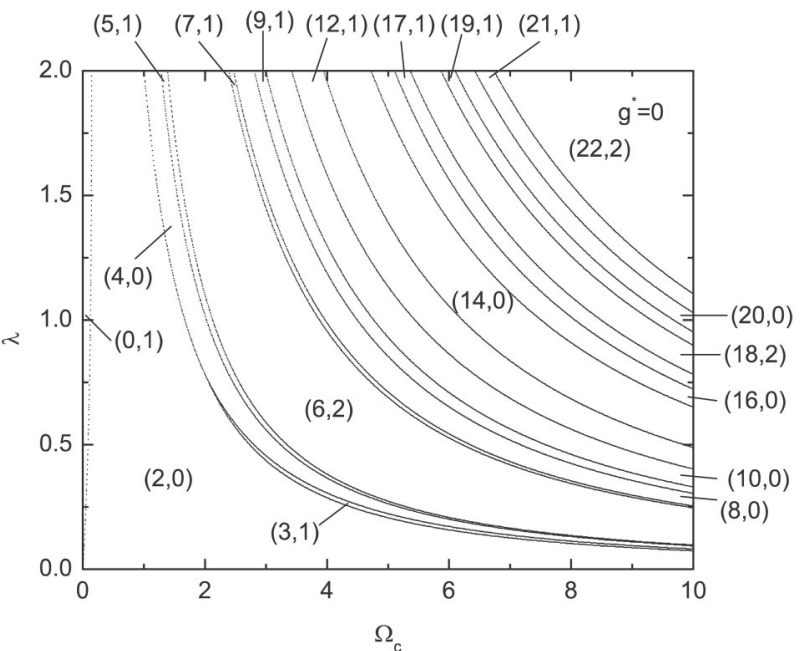

FIG. 3. The ground state configuration as a function of the Coulomb interaction strength and the magnetic field. The Zeeman contribution is not included. Angular momenta up to 22 are shown. States are labeled $(L, S)$ with $L$ the total angular momentum and $S$ the total spin of the state.

four electrons occupy the four lowest orbitals of the lowest LL, i.e., their configuration is $\{(0,0, \uparrow),(0,0, \downarrow),(0,1, \uparrow)$, $(0,1, \downarrow)\}$. The transition from this state to the state $(3,1)$ is accomplished by unpairing the two electrons in the orbital $(0,1, \uparrow \downarrow)$ and promoting one of them to a higher orbital $(0,2, \uparrow)$. This is energetically favorable only when the increase in energy (the distance between these two orbital behaves as $\sim 1 / B$ at strong fields) is balanced by the Zeeman energy (increasing as $\sim B$ ) and exchange energy. If $\lambda$ differs from 0 the transition will occur already at lower magnetic fields, which can be clearly seen from Fig. 2, since the electron repulsion will aid the unpairing of the $(0,1, \uparrow \downarrow)$ electrons. A similar transition happens from the $(3,1)$ to the $(6,2)$ state, but this cannot be seen in Fig. 2. When we compare this transition to the one from the $(6,2)$ state to $(10,2)$ there is a clear difference in mechanism. The latter transition line will approach the $\lambda=0$ line asymptotically, since $(6,2)$ $\rightarrow(10,2)$ transition is only triggered by the electron repulsion.

These considerations lead us to conclude that a similar effect also takes place in quantum-dot lithium, namely, its state $(1,1 / 2)$ is also terminated at a finite magnetic field. However, it was apparently overlooked in Fig. 2(b) of Ref. 15 by not considering sufficiently high magnetic fields.

In general, the inclusion of the Zeeman contribution substantially lowers the energy of the spin-polarized states ( $S$ $=2$ ) at high magnetic fields and leads to the growth of the ground-state angular momentum in steps of 4 , i.e., $6 \rightarrow 10$ $\rightarrow 14 \rightarrow 18 \rightarrow 22$, all of these states being fully polarized. The intermediate partially polarized states $(9,1)$ and $(12,1)$ survive only at the lower end of the magnetic-field range as they are squeezed out by the fully polarized states (see Fig. 2). In contrast, when we neglect the Zeeman term, the partially polarized as well as the unpolarized states survive at high magnetic fields. Therefore, most of the time the ground-state 


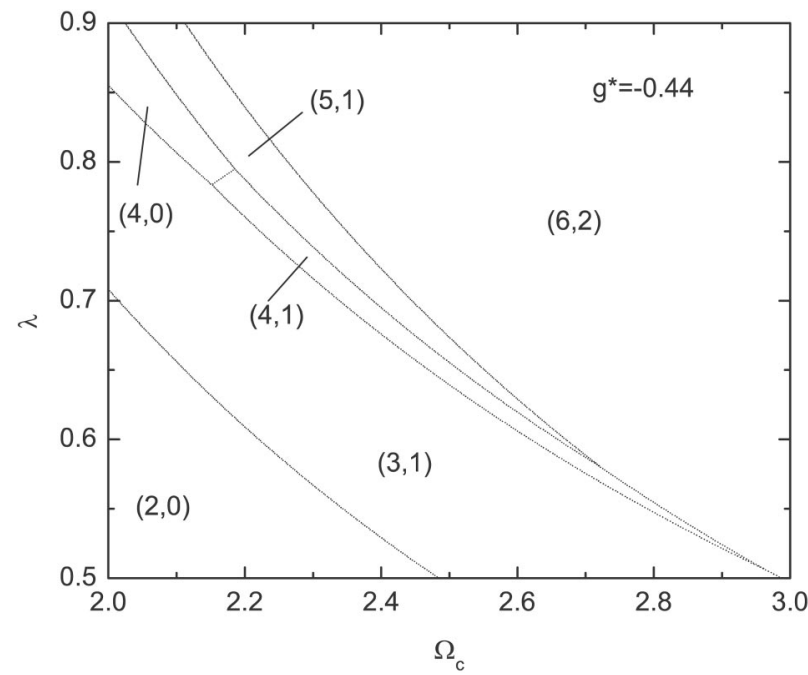

FIG. 4. The magnification of the area indicated by a rectangle in Fig. 2. States are labeled $(L, S)$ with $L$ the total angular momentum and $S$ the total spin of the state.

angular momentum increases in steps of 1 and the angularmomentum transitions are accompanied by corresponding switchings of the spin state.

At intermediate magnetic fields the phase diagram exhibits a rich structure. In Fig. 4 we display a magnification of the region of Fig. 2 marked by the rectangle. One notes that by choosing different fixed values of $\lambda$ and increasing the magnetic field it is possible to perform a transition from the spin-unpolarized MDD state $(2,0)$ to the spin-polarized MDD state $(6,2)$ in six distinct ways. Enumerated in order of increasing $\lambda$ they are

$$
\begin{gathered}
(2,0) \rightarrow(3,1) \rightarrow(6,2), \\
(2,0) \rightarrow(3,1) \rightarrow(4,1) \rightarrow(6,2), \\
(2,0) \rightarrow(3,1) \rightarrow(4,1) \rightarrow(5,1) \rightarrow(6,2), \\
(2,0) \rightarrow(3,1) \rightarrow(4,0) \rightarrow(4,1) \rightarrow(5,1) \rightarrow(6,2), \\
(2,0) \rightarrow(3,1) \rightarrow(4,0) \rightarrow(5,1) \rightarrow(6,2), \\
(2,0) \rightarrow(4,0) \rightarrow(5,1) \rightarrow(6,2) .
\end{gathered}
$$

In particular, in the upper left corner of Fig. 4 we observe a rather peculiar phase boundary between the states $(4,0)$ and $(4,1)$ which was not seen for $g^{*}=0$. At this transition the total electron spin switches from singlet to triplet unaccompanied by an angular-momentum transition. When we increase (in absolute value) the effective $g$ factor to -0.6 (instead of -0.44 as before) we obtain the phase diagram as shown in Fig. 5. The $(4,0)-(4,1)$ transition line has shifted towards lower $\Omega_{c}$ and higher $\lambda$. Decreasing $|g *|$ leads to the opposite behavior. Since the $(4,0)$ state and the $(4,1)$ state differ in total spin it is clear that the Zeeman contribution is essential to make such a transition possible.

This rich structure is a consequence of the interplay between the confinement, Coulomb interaction, and the Zee-

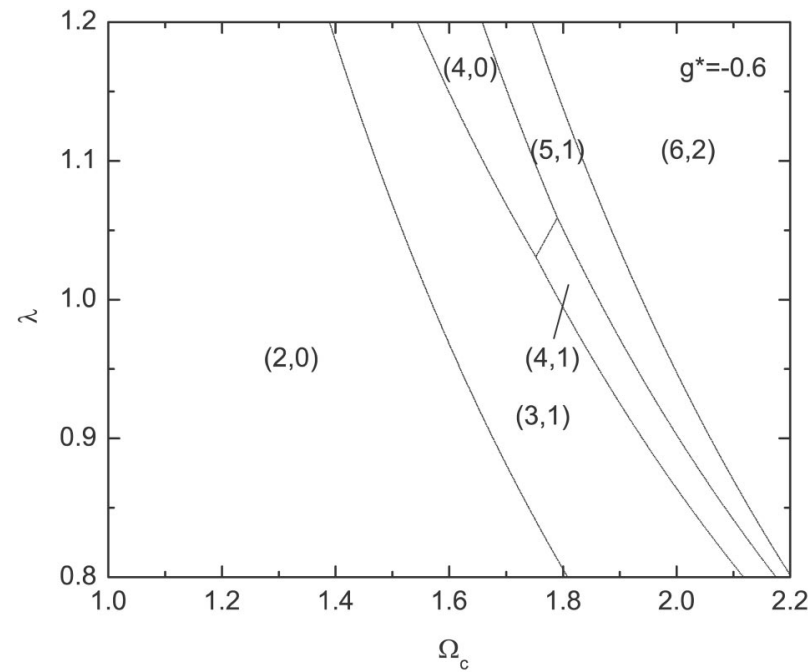

FIG. 5. The same transitions lines as shown in Fig. 4 but now for $g^{*}=-0.6$. States are labeled $(L, S)$ with $L$ the total angular momentum and $S$ the total spin of the state.

man effect. By comparing the $B-\lambda$ phase diagram of our system to that previously obtained for quantum-dot lithium ${ }^{15}$ we may conclude that the addition of an extra electron substantially enriches the phase, and thus we already spot so much interesting physics below $\lambda=2.0$.

\section{B. Wigner crystallization}

In Fig. 6 we show the evolution of the total electron density of the system at $\lambda=2.0$ and varying magnetic field (see also Ref. 13). We see that even at the lowest magnetic fields the density profile has a rather inconspicuous minimum at the center and a maximum at a finite radius which is a con-

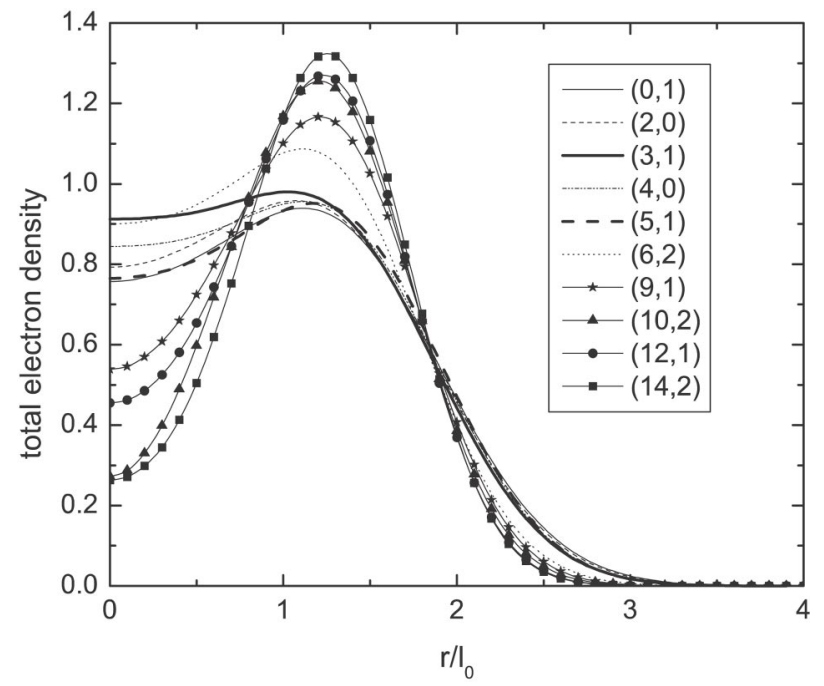

FIG. 6. The total electron density in a number of different angular momentum states at $\lambda=2.0$. These states are the ground states at, respectively, $\Omega_{c}$ equal to $0.0,0.5,1.0,1.1,1.27,2.0,2.7,3.0$, 3.6, and 4.0. Angular momenta up to 14 are included. States are labeled $(L, S)$ with $L$ the total angular momentum and $S$ the total spin of the state. 

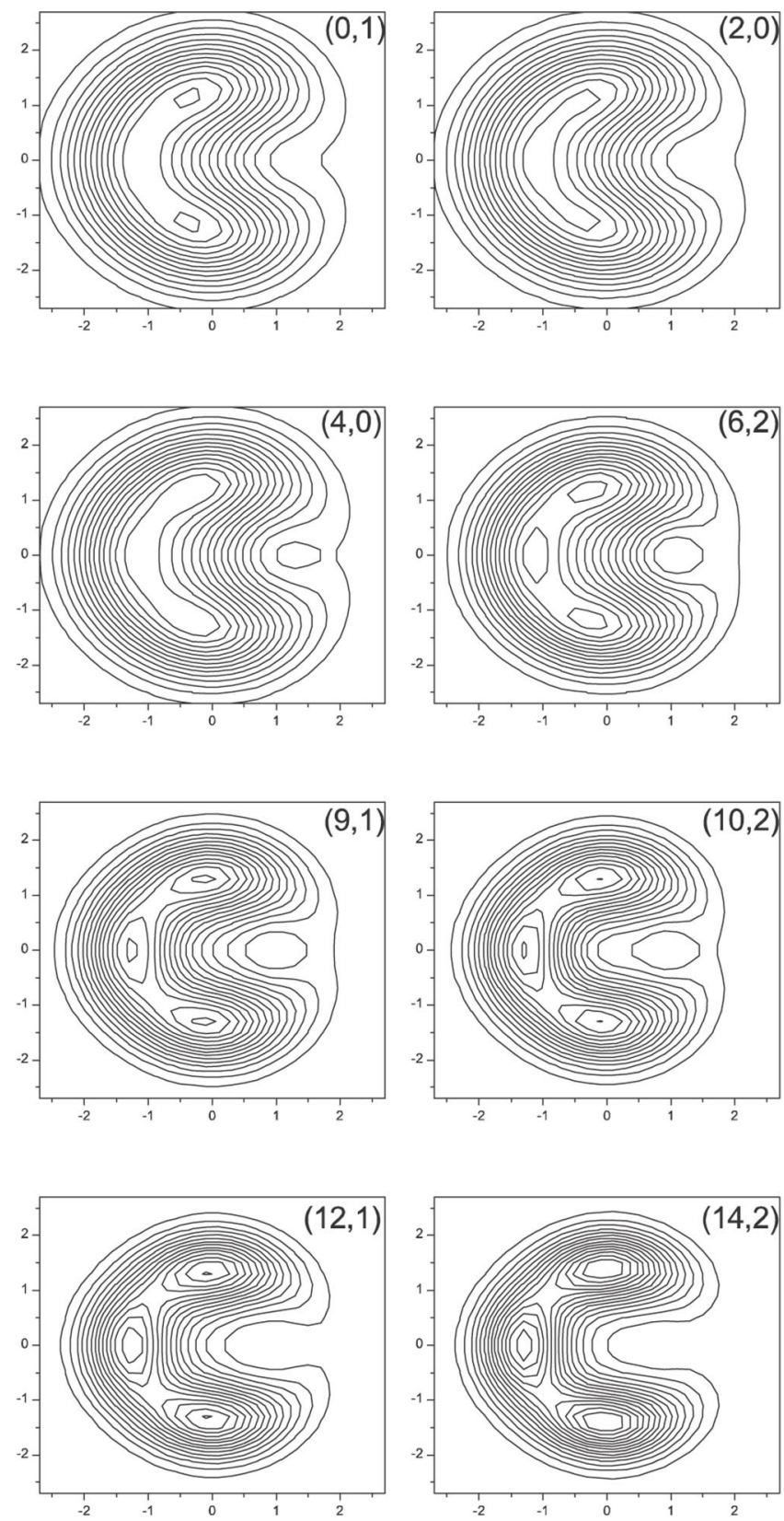

FIG. 7. The total pair-correlation function in a number of different angular momentum states at $\lambda=2.0$. One electron is pinned at $(1.24,0)$. These states are the ground states at, respectively, $\Omega_{c}$ equal to $0.0,0.5,1.1,2.0,2.7,3.0,3.6$, and 4.0. The length is measured in units of $l_{0}$. States are labeled $(L, S)$ with $L$ the total angular momentum and $S$ the total spin of the state.

sequence of a rather high value of $\lambda$. After the breakdown of the spin-polarized MDD state the maximum of the density becomes more pronounced and slightly shifts towards higher radii. This is what we expect from the MDD breakdown since some electrons are forced into higher angular momentum orbits. Thus, increasing magnetic field forces the electrons to be located on a ring, such as for the equivalent classical system with the same radius, which in this case of $\lambda=2.0$ equals 1.24 (see Ref. 16).

We plot the total pair-correlation function in Fig. 7. With

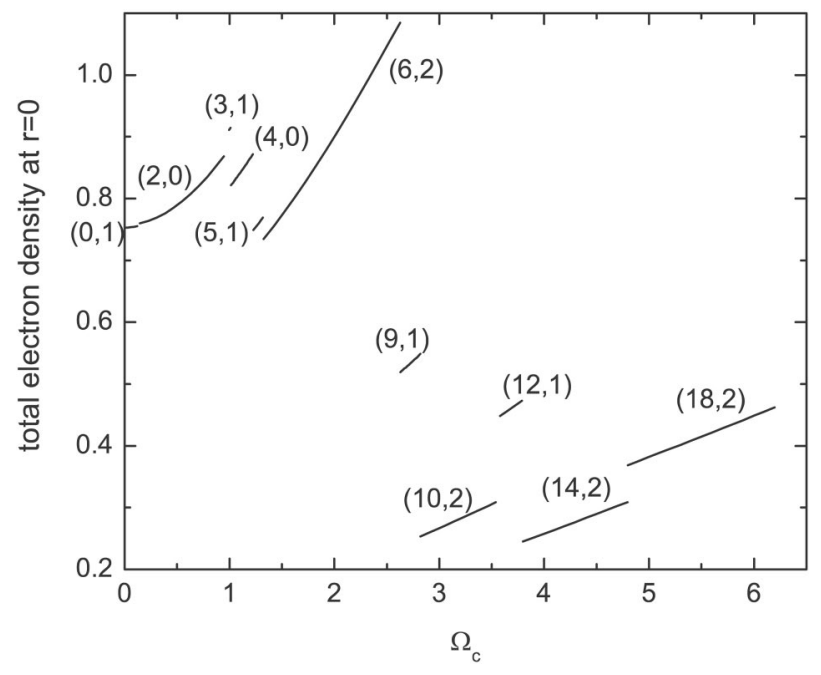

FIG. 8. The total electron density at $r=0$ as a function of the magnetic field at $\lambda=2.0$. Angular momenta up to 18 are included. States are labeled $(L, S)$ with $L$ the total angular momentum and $S$ the total spin of the state.

increasing magnetic field, which induces increasing values of angular momentum, we observe the formation of a Wigner crystal in which the four electrons are located on the corners of a square. From Fig. 8 one can clearly see the high density at $r=0$ for the MDD state and the sudden decrease of the density at the breakdown of the MDD.

Figure 9 shows the behavior of $\left\langle r^{2}\right\rangle$ which, when multiplied by $\pi$, gives an estimate for the size of the dot. At every transition the size of the dot increases discontinuously as the system jumps to a higher angular momentum state. Notice the high compressibility of the spin-polarized MDD state, before it breaks down due to the increasing electron-electron repulsion. The dashed curve in Fig. 9 represents the MCHF

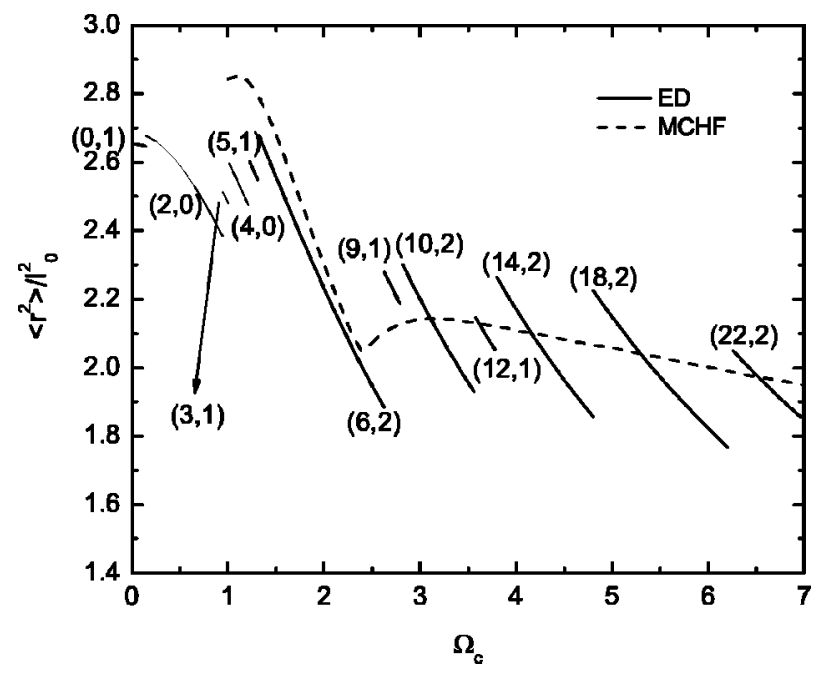

FIG. 9. The squared dot radius $\left\langle r^{2}\right\rangle$ (in units $l_{0}^{2}$ ) as a function of magnetic field $\Omega_{c}$ at $\lambda=2.0$ in the ground state. At each transition of the ground state $\left\langle r^{2}\right\rangle$ increases abruptly. Angular momenta up to 22 are included. The ED and MCHF results are indicated with solid and dashed lines, respectively. States are labeled $(L, S)$ with $L$ the total angular momentum and $S$ the total spin of the state. 


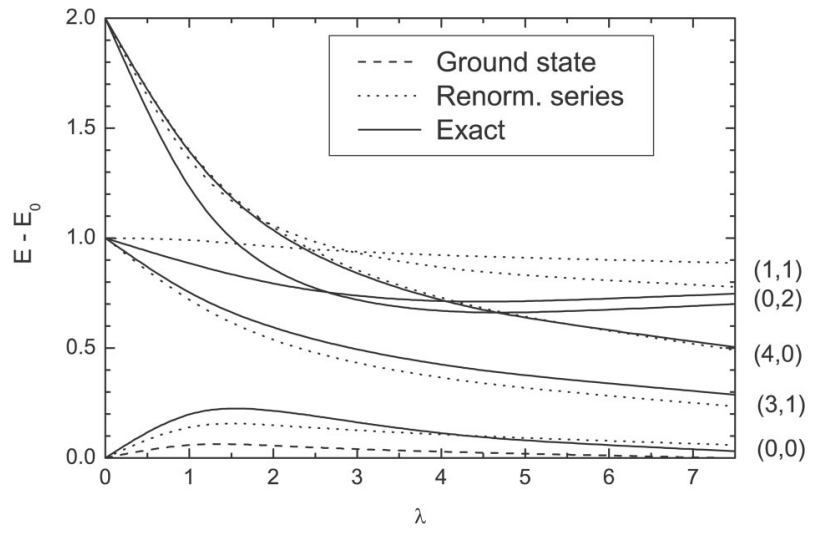

FIG. 10. Excitation energies of several low-lying excited states of the four-electron quantum dot. Differences of the respective state energies and the exact ground-state energy are plotted. Full lines denote exact diagonalization results while dotted lines are obtained from an approximate treatment based on the renormalized perturbation series. Formation of the asymptotic levels is apparent. The dashed line denotes the discrepancy between exact and approximate ground-state energies. States are labeled $(L, S)$ with $L$ the total angular momentum and $S$ the total spin of the state.

result. There is a clear cusp in the dashed curve at $\Omega_{c} \approx 2.4$ which indicates the breakdown of the MDD state, while the actual breakdown of the MDD state as calculated with the ED method happens at $\Omega_{c} \approx 2.6$. After the breakdown of the MDD the MCHF result slightly increases and then starts decreasing towards the classical limit of $\left\langle r^{2}\right\rangle=1.24^{2}=1.538$. For example, at $\Omega_{c}=100\left\langle r^{2}\right\rangle=1.569$. Also in the ED result this decreasing tendency can be observed after the breakdown of the MDD.

\section{COMPARISON TO OTHER METHODS}

\section{A. Excited states}

In the present subsection we consider the excited states of a four-electron quantum dot comparing our results to those obtained previously in Ref. 18. In that paper it was argued that the energy spectra of few-electron quantum dots in a broad range of $\lambda$ values can be rather accurately obtained from an interpolation between few-term expansions valid at $\lambda \rightarrow 0$ and $\lambda \rightarrow \infty$. In particular, it was shown that at high values of $\lambda$ semiclassical vibrational levels are formed. We restrict the present comparison to the case of zero magnetic field since in both methods the finite magnetic field results are obtained by means of rescaling of zero-field results [see Eq. (3) and the foregoing discussion].

In Fig. 10 we show the evolution of a few lowest energy levels from the low- $\lambda$ limit up to $\lambda=7.5$ where our calculations still offer a reasonable accuracy of $\sim 10^{-3}$. Since the absolute energy values grow very rapidly with $\lambda$ we plot their differences from the energy of the ground state $(1,1)$. Thus, the exact ground state energy is identical with 0 in this figure while its approximate value obtained from the interpolation is shown by the dashed line. One sees that the absolute error is always below 0.1 which corresponds to an accuracy better than $1 \%$.
TABLE I. Comparison of the ground state energy for the ED, MCHF, and RHF methods. $g^{*}=-0.44$ and $\lambda=2.0$. Energies are given in units $\hbar \omega_{0}$. The fourth column contains the overestimation of the exact energy obtained with MCHF. The last column contains the overestimation of the exact energy obtained with RHF.

\begin{tabular}{cccccc}
\hline \hline$\Omega_{c}$ & ED & MCHF & $\Delta E_{\mathrm{MCHF}}$ & RHF & $\Delta E_{\mathrm{RCHF}}$ \\
\hline 1.892 & 14.815 & 15.026 & 0.211 & 14.933 & 0.118 \\
3.154 & 16.846 & 17.046 & 0.200 & 17.095 & 0.249 \\
4.416 & 19.010 & 19.201 & 0.191 & 19.444 & 0.434 \\
5.678 & 21.258 & 21.450 & 0.192 & 21.859 & 0.601 \\
6.939 & 23.610 & 23.764 & 0.154 & 24.302 & 0.692 \\
8.201 & 26.043 & 26.125 & 0.082 & 26.761 & 0.718 \\
9.462 & 28.441 & 28.516 & 0.075 & 29.226 & 0.785 \\
\hline \hline
\end{tabular}

The three states $(0,0),(3,1)$, and $(4,0)$ all tend to the lowest vibrational level in the $\lambda \rightarrow \infty$ limit. We see that for the state $(4,0)$ the coincidence of the two results is surprisingly accurate and the difference between the two lines is hard to see on the plot. In contrast to this, the mismatch between the exact and the approximate results for the states $(0,2)$ and $(1,1)$, which both tend to the first excited asymptotic vibrational level, is considerably larger, especially in the intermediate region $2<\lambda<5$. However, at higher values of $\lambda$ the accuracy improves and it is apparent that the levels $(0,2)$ and $(1,1)$ indeed form a distinct group separating from the other terms described earlier.

\section{B. Ground state energy}

In Table I we compare the results of the present ED to those of the MCHF and RHF methods for the ground state energy of the system as a function of $\Omega_{c}$ at $\lambda=2.0$. At low magnetic fields the ED yields lower (and correct) values for the energy. When the magnetic field increases the MCHF approaches the ED result, since the system becomes more classical, whereas the RHF result diverges from the ED result. This can be understood from the fact that the RHF method does not take correlation effects into account while apparently these effects become more and more important after the breakdown of the MDD. One notes that the convergence of the MCHF to the ED result is not monotonous. This is a consequence of the small cusps which appear in the ED result (see Fig. 1) when a ground state transition occurs while the MCHF result is a smooth curve as a function of the magnetic field.

\section{Phase diagram}

Figures 2 and 3 show that the spin-angular momentum configuration of the four-electron ground state is a complex function of the strength of the electron-electron interaction and the external magnetic field. We have compared the exact phase diagrams with the results obtained with the RHF (the MCHF solutions do not possess a definite angular momentum).

For $g^{*}=0$ and $\lambda=0.5$ (see Fig. 3) the RHF method correctly predicts the ground state for zero magnetic field to 
have $(0,1)$ symmetry. However, the critical magnetic fields for the transformation of the ground state to $(2,0)$ is overestimated by about $50 \%\left(\Omega_{c}^{\mathrm{RHF}}=0.112\right.$, instead of the exact value $\left.\Omega_{c}=0.085\right)$. On the other hand, according to RHF the ground state changes from $(2,0)$ to $(3,1)$ for $\Omega_{c}^{\mathrm{RHF}}=2.54$, while the exact value is 2.73 . According to RHF state $(3,1)$ is transformed to the MDD $(6,2)$ at higher magnetic field $\left(\Omega_{c}^{\mathrm{RHF}}=2.99\right)$. In contrast to the exact calculations the RHF does not predict the $(4,0)$ and $(5,1)$ states to be ground states. In the exact calculations the MDD becomes the ground state for $\Omega_{c}^{\mathrm{RHF}}=3.34$.

The difference in the estimation of the critical magnetic fields reveals the known tendency of the RHF to overestimate the exchange interaction and to favor spin-polarized states, for which the precision of the method is better than for unpolarized states. We notice that the values of $\Omega_{c}$ inducing the ground-state transformations to higher-spin states are underestimated, while the values of $\Omega_{c}$ for which the spin of the ground state is decreased are overestimated by the RHF method.

After the MDD decay for $g^{*}=0$ the exact calculations predict a sequence of the states not necessarily spin polarized (see Fig. 3). On the other hand, the RHF after the MDD decay predicts only the appearance of the spin-polarized ground states. Moreover, according to RHF only the states of the magic sequence $(6+4 n, 2)$ become ground states at high magnetic field. Such magic sequence in the exact solution is observed only for nonzero $g$ factor (see Fig. 2). In this sense the error of the RHF introduces an "internal $g$ factor" to the electron system.

Results of the energy presented in Table I indicate that the correlation energy rapidly grows after the MDD breakdown and becomes particularly large when the Wigner crystallization appears. The spin polarization predicted by the RHF is a reflection of the exact effect related with the Wigner crystallization, i.e., the separation of the electron charges. In the RHF method this separation is partially realized only for spin-polarized states, for which probability of finding two electrons in the same place is 0 due to the antisymmetry of the spatial wave function.

Let us consider also the case of larger interaction strength $\left(\lambda=2.0\right.$, see Fig. 3). For $g^{*}=0$ and $\lambda=2.0$ the RHF method predicts that the ground state changes from $(0,1)$ to $(3,1)$ and then to MDD. In this way RHF overlooks the fact that the spin-unpolarized states $(2,0),(4,0)$, and partially polarized $(5,1)$ state are the ground states for a certain range of the magnetic field (see Fig. 3) below the formation of the MDD. According to RHF the fully spin-polarized MDD appears already for $\Omega_{c}^{\mathrm{RHF}}=0.71$, while according to the exact calculations MDD appears in a much larger magnetic field $\Omega_{c}$ $=1.39$. In larger magnetic field RHF still predicts the magic sequence of the states as in the case of $\lambda=0.5$. The results of the RHF for the phase diagrams are qualitatively the same for nonzero $g$ factor.

The present comparison of the phase transitions and the results of the energy presented in Table I shows that the precision of the RHF method deteriorates for strong electronelectron interaction as well as for high magnetic fields. In both cases the electron system is strongly correlated and the Wigner crystallization appears. For nonzero $g$ the RHF gives a qualitatively correct sequence of the ground states after the MDD breakdown, but as we have shown this agreement is rather accidental.

\section{CONCLUSIONS}

To conclude, we obtained the energy spectrum of the fourelectron quantum dot as a function of the magnetic field and we compared it to previously obtained results. We see that a simple few term expansion of Ref. 18 is not fully capable to describe the behavior of excited states in the region intermediate between the perturbative and asymptotic regimes. On the other hand, it is quite remarkable that an exactdiagonalization scheme starting from energy levels of noninteracting electrons is capable of reaching the strong electron interaction limit and revealing the formation of semiclassical energy levels.

A phase diagram has been composed for different values of $g^{*}$. At small magnetic fields we observed the depolarization of spins from the $(0,1)$ to the $(2,0)$ state by application of a magnetic field. A rich structure has been observed at intermediate magnetic fields with a somewhat strange looking transition between the $(4,0)$ and the $(4,1)$ state in which the total spin changes while the angular momentum remains the same. Other transitions have been investigated and their mechanism was explained. We observed the formation of a Wigner crystal by increasing the magnetic field. The sudden decrease of the density at $r=0$ after the breakdown of the MDD state $(6,2)$ shows that this state is special.

Comparison to results obtained with the RHF learns us that this system is highly correlated towards high electronelectron interaction strengths and high magnetic fields. The RHF is only useful in the low correlation regime, e.g., it predicts the right ground state configuration at zero magnetic field. The comparison of the phase diagrams clearly shows the tendency of the RHF to favor polarized states. The $\mathrm{MCHF}$ is proven to be very useful in high magnetic fields since its results converge to the exact results with increasing magnetic field, e.g., at $\Omega_{c}=9.462$ the overestimate of the ground state energy only amounts to 0.075 .

\section{ACKNOWLEDGMENTS}

This work was supported by the European Commission GROWTH program NANOMAT project under Contract No. GSRD-CT-2001-00545, the Belgian Interuniversity Attraction Poles (IUAP), the Flemish Science Foundation (FWOVI), and the Flemish Concerted Action (GOA) programs. E.A. is supported by EU under Contract No. HPMF-CT2001-01195 and B.S. is supported by the Foundation for Polish Science (FNP). Useful discussions with Dr. B. Partoens are acknowledged. 
*Electronic address: maarten.tavernier@ua.ac.be

†Electronic address: egidijus@ua.ac.be

†Electronic address: francois.peeters@ua.ac.be

${ }^{1}$ P.A. Maksym, H. Imamura, G.P. Mallon, and H. Aoki, J. Phys.: Condens. Matter 12, 299 (2000).

${ }^{2}$ L.P. Kouwenhoven, D.G. Austing, and S. Tarucha, Rep. Prog. Phys. 64, 701 (2001).

${ }^{3}$ S.M. Reimann and M. Manninen, Rev. Mod. Phys. 74, 1283 (2002)

${ }^{4}$ S. Bednarek, B. Szafran, and J. Adamowski, Phys. Rev. B 64, 195303 (2001).

${ }^{5}$ A. Matulis and F.M. Peeters, Solid State Commun. 117, 655 (2001).

${ }^{6}$ S.M. Reimann, M. Koskinen, and M. Manninen, Phys. Rev. B 62, 8108 (2000).

${ }^{7}$ M. Wagner, U. Merkt, and A.V. Chaplik, Phys. Rev. B 45, 1951 (1992).

${ }^{8}$ P.A. Maksym, Phys. Rev. B 53, 10871 (1996).

${ }^{9}$ U. Merkt, J. Huser, and M. Wagner, Phys. Rev. B 43, 7320 (1991).

${ }^{10}$ S.-R.E. Yang, A.H. MacDonald, and M.D. Johnson, Phys. Rev. Lett. 71, 3194 (1993).
${ }^{11}$ M. Eto, Jpn. J. Appl. Phys. 36, 3924 (1997).

${ }^{12}$ S.-R.E. Yang and A.H. MacDonald, Phys. Rev. B 66, 041304 (2002).

${ }^{13}$ M. Eto, Jpn. J. Appl. Phys. 38, 376 (1999).

${ }^{14}$ S.A. Mikhailov, Phys. Rev. B 65, 115312 (2001).

${ }^{15}$ S.A. Mikhailov and N.A. Savostianova, Phys. Rev. B 66, 033307 (2002).

${ }^{16}$ S.A. Mikhailov, Phys. Rev. B 66, 153313 (2002).

${ }^{17}$ A. Matulis and F.M. Peeters, J. Phys.: Condens. Matter 6, 7751 (1994).

${ }^{18}$ E. Anisimovas and A. Matulis, J. Phys.: Condens. Matter 10, 601 (1998).

${ }^{19}$ B. Szafran, S. Bednarek, and J. Adamowski, Phys. Rev. B 67, 045311 (2003).

${ }^{20}$ V.M. Bedanov and F.M. Peeters, Phys. Rev. B 49, 2667 (1994).

${ }^{21}$ W.Y. Ruan, Y.Y. Liu, C.G. Bao, and Z.Q. Zhang, Phys. Rev. B 51, 7942 (1994).

${ }^{22}$ S. Tarucha, D.G. Austing, T. Honda, R.J. van der Hage, and L.P. Kouwenhoven, Phys. Rev. Lett. 77, 3613 (1996).

${ }^{23}$ L.P. Kouwenhoven, T.H. Oosterkamp, M.W.S. Danoesastro, M. Eto, D.G. Austing, T. Honda, and S. Tarucha, Science 278, 1788 (1997). 